\title{
Interação e eficácia de misturas em tanque dos herbicidas metsulfuron-methyl e glyphosate
}

\author{
Interaction and efficacy of metusulfuron-methyl and glyphosate \\ tank mixtures
}

\begin{abstract}
Jeisiane de Fátima Andrade ${ }^{1}$, Jéssica Cursino Presoto ${ }^{1}$, Acácio Gonçalves Netto², Saul Jorge Pinto de Carvalho ${ }^{1}$
\end{abstract}

Resumo - A solução mais comum para as falhas no controle de plantas daninhas no sistema de produção é a inclusão de herbicidas alternativos, aplicados de forma isolada ou misturados em tanque. Este trabalho foi desenvolvido com o objetivo de avaliar a eficácia e a interação de misturas de metsulfuron-methyl e glyphosate para controle de plantas daninhas. Para tanto, foram desenvolvidos dois experimentos independentes, avaliando-se diferentes bioindicadores, uma monocotiledônea (capim-amargoso - Digitaria insularis) e uma dicotiledônea (corda-de-viola - Ipomoea triloba). Os tratamentos constaram de esquema fatorial $5 \times 5$, correspondendo o primeiro fator às cinco doses do herbicida glyphosate $\left(0,180,360,540\right.$ e $720 \mathrm{~g}$ e.a. ha $\left.{ }^{-1}\right)$ e o segundo fator às cinco doses do herbicida metsulfuron-methyl $(0 ; 1,2 ; 2,4 ; 3,6$ e 4,8 g i.a. ha-1). Em ambos os experimentos se adotou delineamento experimental de blocos ao acaso, com seis repetições. A aplicação da mistura de glyphosate e metsulfuron-methyl sobre o capim-amargoso foi considerada aditiva em todas as doses utilizadas; já sobre a corda-de-viola, a adição de metsulfuron-methyl à calda de glyphosate eleva o controle absoluto da mistura, porém com magnitude aquém da esperada, o que denota caráter antagônico.

Palavras chave: plantas daninhas, manejo, resistência, tolerância, sinergia, antagonismo

\begin{abstract}
The most common solution for problems on weed control in agricultural system of production is the inclusion of alternative herbicides, applied isolated or in tank mixtures. Thus, this work was carried out with the objective of evaluating the efficacy and interaction of metsulfuron-methyl and glyphosate tank mixtures for weed control. For that, two independent trials were performed, evaluating different bioindicators, being one monocotyledonous (sourgrass - Digitaria insularis) and one dicotyledonous (morning glory - Ipomoea triloba). Treatments were consequence of a factorial combination 5x5, corresponding the first factor to five rates of glyphosate $\left(0,180,360,540\right.$ and $720 \mathrm{~g}$ a.e. ha $\left.\mathrm{ha}^{-1}\right)$ and the second factor to five rates of metsulfuron-methyl $\left(0 ; 1.2 ; 2.4 ; 3.6\right.$ and $\left.4.8 \mathrm{~g} \mathrm{a.i.} \mathrm{ha}^{-1}\right)$. In both experiments, randomized blocks were adopted as experimental design, with six replicates.
\end{abstract}

\footnotetext{
Recebido: Julho 09, 2018. Aceito: Fevereiro 13, 2019.

${ }^{1}$ Instituto Federal de Educação, Ciência e Tecnologia do Sul de Minas Gerais - IFSULDEMINAS, Campus Machado, Rod. Machado-Paraguaçu, km. 3, Bairro Santo Antonio, 37750-000, Machado, MG, Brasil. E-mail: jeisiane.eng.agronomia@gmail.com; jessica.cursino_02@hotmail.com; sjpcarvalho@yahoo.com.br

2 Escola Superior de Agricultura "Luiz de Queiroz" - ESALQ/USP, Av. Pádua Dias, 11, CP. 09, 13418-900, Piracicaba, SP, Brasil. E-mail: acaciogn@agronomo.eng.br
} 
The mixture of glyphosate and metsulfuron-methyl was considered additive on sourgrass for all the rates considered; for morning glory, including metsulturon-methyl on tank mixture increased total control, however with magnitude lower than expected, what denotes antagonist behavior of mixture. Keywords: weeds, management, resistance, tolerance, synergy, antagonism

\section{Introdução}

O Brasil possui grande potencial de produção agrícola devido as condições edafoclimáticas favoráveis aos cultivos, entretanto existe uma série de fatores que podem limitar a produtividade das culturas. Dentre estes fatores, as plantas daninhas possuem destaque no sistema agrícola por causarem decréscimos na produtividade das culturas e aumento nos custos de produção, o que torna necessária a adoção dos herbicidas como a principal estratégia de controle da comunidade infestante (Ciuberkis et al., 2010).

Dentre os herbicidas utilizados para o controle de plantas daninhas destaca-se o glyphosate como a principal molécula adotada (Carbonari et al., 2005; Moldes et al., 2012). O glyphosate age como um herbicida não seletivo e de amplo espectro de ação, tendo como mecanismo de ação a inibição da enzima 5-enolpiruvil-chiquimato-3-fosfato-sintase (EPSPs), levando ao bloqueio da rota do ácido chiquímico e consequentemente a síntese de aminoácidos e metabólitos secundários (Moldes et al., 2012).

O aumento de sistemas de produção conservacionistas (plantio direto) e a maior flexibilidade para aplicação do produto em culturas geneticamente modificadas (transgênicos) aliado ao uso constante do mesmo ingrediente ativo resultaram na seleção de biótipos de plantas daninhas resistentes ao glyphosate, bem como de espécies tolerantes a este herbicida, por consequência da maior pressão de seleção imposta, relacionada ao maior número de aplicações do produto (Christoffoleti et al., 2008).

Segundo Peterson (1999), quando a resistência ou tolerância é detectada em uma área agrícola, a mudança mais comum adotada pelos agricultores no sistema produtivo é a inclusão de moléculas com diferentes mecanismos de ação no sistema. Isto pode ser feito com a inclusão de herbicidas alternativos, aplicados de forma isolada ou por meio de misturas em tanque com aqueles herbicidas para os quais a resistência ou tolerância foi detectada.

Assim, é importante associar herbicidas com diferentes mecanismos de ação ao glyphosate como estratégia de controle de plantas daninhas resistentes ou tolerantes (Ramires et al., 2010; Maciel et al., 2011), ou mesmo como medida preventiva para retardar o aparecimento da resistência. Uma vez que se inclui um herbicida com mecanismo de ação diferente daquele para o qual a resistência ou tolerância foi identificada, além de alterar o mecanismo de controle, a frequência de seleção de biótipos resistentes é significativamente reduzida.

Dentre os herbicidas com uso frequente em associação com o glyphosate, particularmente para manutenção em culturas perenes (citrus e café), destaca-se o metsulfuron-methyl, herbicida inibidor da enzima acetolactato sintase (ALS) (Alcântara e Silva, 2002; Moreira et al., 2010). Embora o uso de misturas de herbicidas seja muito comum na agricultura brasileira, há pouca disponibilidade de informações sobre a comparação da eficácia e interação dos herbicidas envolvidos, os quais podem responder de forma sinérgica, aditiva ou antagônica (Gazziero, 2015).

Quando o controle obtido com uma mistura é maior que a simples combinação dos controles dos herbicidas aplicados isolados (controle esperado) considera-se seu efeito sinérgico; se o controle obtido é menor do que o controle esperado da mistura, considera-se como efeito antagônico. Quando o controle alcançado pela mistura é equivalente ao controle esperado, a resposta é considerada aditiva (Lich et al., 1997; Gazziero, 
2015). Desta forma, este trabalho foi desenvolvido com o objetivo de avaliar a interação das misturas de glyphosate e metsulfuron-methyl e sua eficácia para o controle de plantas daninhas.

\section{Material e métodos}

Dois experimentos foram desenvolvidos em casa-de-vegetação do Instituto Federal de Educação, Ciência e Tecnologia do Sul de Minas Gerais, Câmpus Machado - MG (21 ${ }^{\circ} 40^{\prime}$ $\mathrm{S} ; 45^{\circ} 55^{\prime} \mathrm{W}$; $850 \mathrm{~m}$ de altitude), durante o ano de 2015. Em cada experimento adotou-se uma espécie bioindicadora distinta, utilizando-se de uma monocotiledônea (Digitaria insularis capim-amargoso, suscetível ao glyphosate) e uma dicotiledônea (Ipomoea triloba - corda-de-viola), ambas consideradas de difícil controle. Estas espécies foram adotadas no intuito de avaliar a interferência da mistura sobre a eficácia do glyphosate (controle de D. insularis), bem como sobre o metsulfuron-methyl (controle de I. triloba).

As sementes dos bioindicadores foram germinadas em caixas plásticas com capacidade para $2.000 \mathrm{~mL}$, preenchidas com substrato comercial (casca de Pinus + turfa + vermiculita). Em estádio fenológico de uma folha plenamente expandida (monocotiledôneas) ou em folhas cotiledonares (dicotiledôneas), as plântulas foram transplantadas para vasos onde permaneceram até o final do experimento, em população média de oito plantas por vaso (D. insularis) ou três plantas por vaso (I. triloba).

Os experimentos foram instalados seguindo o modelo de delineamento experimental de blocos ao acaso, em que cada parcela constou de um vaso com capacidade para 1,0 L, preenchido com a mistura de substrato comercial, terra peneirada e vermiculita na proporção de 6:3:1, respectivamente. Todas as parcelas foram devidamente fertilizadas e irrigadas diariamente. Cada experimento contou com arranjo fatorial $5 \times 5$, sendo os níveis do primeiro fator as doses de glyphosate $\left(0,180,360,540\right.$ e 720 g e.a. ha $\left.{ }^{-1}\right)$ e os níveis do segundo fator as doses do metsulfuron-methyl
$(0 ; 1,2 ; 2,4 ; 3,6$ e 4,8 g i.a. ha-1 $)$, resultando em 25 tratamentos e 150 parcelas (seis repetições). Em todos os tratamentos, utilizou-se água deionizada no preparo das soluções, para evitar contaminação.

As pulverizações foram realizadas 40 dias após semeadura, sobre plantas em pré-perfilhamento (D. insularis) ou quatro folhas plenamente expandidas (I. triloba). Para tanto, foi utilizado pulverizador costal de precisão, pressurizado por $\mathrm{CO}_{2}$, acoplado a uma única ponta do tipo TeeJet XR 110.02, posicionada a $0,50 \mathrm{~m}$ dos alvos e volume de calda de $200 \mathrm{~L} \mathrm{ha}^{-1}$.

Posteriormente, avaliou-se o controle percentual aos 14 e 28 dias após aplicação (DAA), bem como a massa seca residual aos 28 DAA. Para as avaliações de controle percentual, foi atribuído $0 \%$ no caso da ausência de sintomas causados pelos herbicidas e $100 \%$ para a morte das plantas, segundo o método proposto pela Sociedade Brasileira da Ciência das Plantas Daninhas (SBCPD, 1995). A massa vegetal se obteve a partir da colheita do material vegetal remanescente nas parcelas, com posterior secagem em estufa de circulação forçada a $70{ }^{\circ} \mathrm{C}$ por 72 horas.

A análise dos dados foi realizada por meio da aplicação do teste $\mathrm{F}$ na análise da variância, seguido do emprego do teste de Scott-Knott, ambos com 5\% de significância. Se considerado apenas um nível de tratamentos, observam-se tratamentos quantitativos, porém para viabilizar a análise de antagonismo-sinergia das misturas não foram aplicadas regressões.

A análise de antagonismo-sinergia foi baseada no modelo proposto por Colby (1967):

$$
\mathrm{E}=100-\frac{(100-\mathrm{X}) *(100-\mathrm{Y})}{100}
$$

Em que: $X$ é a porcentagem de inibição do crescimento (controle) pelo glyphosate na dose $x$; $Y$ é a porcentagem de inibição do crescimento (controle) pelo herbicida metsulfuron-methyl na dose $y$; e $E$ é a porcentagem esperada da 
mistura dos herbicidas na mesma dose $(x+y)$ (Barroso et al., 2014; Trezzi et al., 2016).

Então, $E$ pode ser entendida como a toxicidade esperada da mistura. Se a resposta observada for maior que a esperada, a mistura é sinérgica; se a resposta observada for menor que a esperada, a mistura é antagônica; se a resposta observada for igual à esperada, a mistura é aditiva. Para comparação da resposta esperada e resposta observada adotou-se o teste ' $\mathrm{t}$ ', com $5 \%$ de significância.

\section{Resultados e discussão}

Em relação ao controle do capim-amargoso, em todas as avaliações evidenciou-se interação das misturas de glyphosate e metsulfuron-methyl (Tabela 1). Notadamente, apenas foram alcançados níveis satisfatórios no controle do capim-amargoso, superiores a $80 \%$, com o incremento da dose de glyphosate, não havendo diferenças com o incremento na dose de metsulfuron-methyl. Neste caso, viabilizou-se a análise da interferência da mistura sobre a eficácia do glyphosate.

O glyphosate é um herbicida não seletivo que possui amplo espectro de controle (Rodrigues e Almeida, 2011), o que se confirmou nos resultados de controle percentual aos 28 DAA, que evidenciaram sua elevada eficácia sobre o capim-amargoso em doses superiores a $540 \mathrm{~g} \mathrm{ha}^{-1}$ (Tabela 1). Os sintomas observados estão em concordância com a literatura que registra que as plantas tratadas se desenvolvem lentamente, manifestando cloroses nas regiões meristemáticas ou nas folhas mais jovens, seguidas de necrose foliar entre uma ou três semanas após a aplicação, de acordo com a suscetibilidade da espécie. A folhagem por vezes torna-se roxo-avermelhada em algumas espécies (Vidal et al., 2014). Outros sintomas foliares comuns são: enrugamento ou más-formações (especialmente nas áreas de rebrotamento) assim como necrose de meristemas e também de rizomas e estolões de plantas perenes (Vargas et al., 2006).

Tabela 1. Controle percentual ${ }^{1}$ do capim-amargoso (Digitaria insularis) após pulverização de diferentes doses do herbicida glyphosate e metsulfuron-methyl, isoladas ou em mistura, avaliados aos 14 e 28 DAA. Machado - MG, 2015.

\begin{tabular}{|c|c|c|c|c|c|}
\hline \multirow{2}{*}{$\begin{array}{c}\text { Glyphosate } \\
\left(\mathrm{g} \mathrm{ha}^{-1}\right)\end{array}$} & \multicolumn{5}{|c|}{ Metsulfuron-methyl (g ha-1) } \\
\hline & $\mathbf{0}$ & 1,2 & 2,4 & 3,6 & 4,8 \\
\hline \multicolumn{6}{|c|}{ Avaliação de controle realizada aos 14 dias após aplicação } \\
\hline 0 & $0,0 \mathrm{C} \mathrm{b}$ & $18,0 \mathrm{D}$ a & $15,3 \mathrm{~B} \mathrm{a}$ & $25,8 \mathrm{~B} \mathrm{a}$ & $18,3 \mathrm{~B} \mathrm{a}$ \\
\hline 180 & $28,7 \mathrm{~B} \mathrm{~b}$ & $36,7 \mathrm{C} \mathrm{a}$ & $19,2 \mathrm{~B} \mathrm{~b}$ & $35,8 \mathrm{~B}$ a & $49,7 \mathrm{~A} \mathrm{a}$ \\
\hline 360 & $42,5 \mathrm{~A} \mathrm{a}$ & $37,8 \mathrm{C} \mathrm{a}$ & $49,2 \mathrm{~A} \mathrm{a}$ & $43,2 \mathrm{~B}$ a & $58,7 \mathrm{~A} \mathrm{a}$ \\
\hline 540 & $50,5 \mathrm{~A} \mathrm{a}$ & $52,2 \mathrm{~B}$ a & $64,3 \mathrm{~A} \mathrm{a}$ & $37,2 \mathrm{~B}$ a & $47,3 \mathrm{~A} \mathrm{a}$ \\
\hline \multirow[t]{2}{*}{720} & $55,7 \mathrm{~A} \mathrm{a}$ & $75,0 \mathrm{~A} \mathrm{a}$ & $61,3 \mathrm{~A} \mathrm{a}$ & $66,7 \mathrm{~A} \mathrm{a}$ & $67,8 \mathrm{~A} \mathrm{a}$ \\
\hline & $\mathrm{CV}=21,57$ & $\mathrm{~F}_{\mathrm{int}}=2,651 * *$ & $\mathrm{~F}_{\mathrm{gly}}=46,244 * *$ & $\mathrm{~F}_{\mathrm{met}}=3,903 * *$ & \\
\hline \multicolumn{6}{|c|}{ Avaliação de controle realizada aos 28 dias após aplicação } \\
\hline 0 & $0,0 \mathrm{D} \mathrm{b}$ & $14,7 \mathrm{C} \mathrm{a}$ & $10,8 \mathrm{C} \mathrm{a}$ & $22,2 \mathrm{~B} \mathrm{a}$ & $15,8 \mathrm{C} \mathrm{a}$ \\
\hline 180 & $36,3 \mathrm{C} \mathrm{b}$ & $51,3 \mathrm{~B}$ a & $29,8 \mathrm{~B} \mathrm{~b}$ & $39,3 \mathrm{~B} \mathrm{~b}$ & $65,8 \mathrm{~B} \mathrm{a}$ \\
\hline 360 & $66,0 \mathrm{~B} \mathrm{a}$ & $53,3 \mathrm{~B} \mathrm{a}$ & $82,2 \mathrm{~A} \mathrm{a}$ & $76,3 \mathrm{~A} \mathrm{a}$ & $76,5 \mathrm{~B}$ a \\
\hline 540 & $91,3 \mathrm{~A} \mathrm{a}$ & $88,2 \mathrm{~A} \mathrm{a}$ & $95,5 \mathrm{~A} \mathrm{a}$ & $88,2 \mathrm{~A} \mathrm{a}$ & $88,2 \mathrm{~A} \mathrm{a}$ \\
\hline \multirow[t]{2}{*}{720} & $95,8 \mathrm{~A} \mathrm{a}$ & $99,5 \mathrm{~A} \mathrm{a}$ & $97,2 \mathrm{~A} \mathrm{a}$ & $98,0 \mathrm{~A}$ a & $98,2 \mathrm{~A} \mathrm{a}$ \\
\hline & $\mathrm{CV}=17,05$ & $\mathrm{~F}_{\mathrm{int}}=2,617^{* *}$ & $\mathrm{~F}_{\mathrm{gly}}=137,377^{* *}$ & $\mathrm{~F}_{\mathrm{met}}=2,962^{*}$ & \\
\hline
\end{tabular}

${ }^{1}$ Médias seguidas por letras iguais, maiúsculas nas colunas e minúsculas nas linhas, não diferem entre si segundo teste de Scott-Knott, com 5\% de significância. *Teste F significativo a 5\%; **Teste F significativo a 1\% de probabilidade. 
O herbicida metsulfuron-methyl possui moderada eficácia no controle de espécies monocotiledôneas (Rodrigues e Almeida, 2011; Vidal et al., 2014), o que se evidenciou aos 14 e 28 DAA onde as doses isoladas do mesmo não foram capazes de alcançar controle satisfatório do capim-amargoso (Tabela 1). Para avaliação de massa seca, não foi identificada interação, obtendo-se somente o efeito das diferentes doses de glyphosate (Tabela 2). Novamente, apenas foram alcançados níveis satisfatórios no controle do capim-amargoso com o incremento da dose de glyphosate, não havendo diferenças com o incremento na dose de metsulfuron-methyl. Desta forma, foi possível avaliar o efeito da mistura sobre a eficácia do herbicida glyphosate, visto que foi este produto que efetivamente promoveu o controle do capim-amargoso (Tabelas 1 e 2).

Adotando-se a corda-de-viola como espécie bioindicadora, em nenhuma das avaliações obteve-se controle percentual considerado satisfatório, da ordem de $80 \%$ (Tabela 3 ). Porém, neste caso, além da variável controle, também foi detectada interação fatorial para a variável massa de matéria seca (Tabela 4), em contraponto ao que foi identificado para o capim-amargoso.

Neste caso, observou-se maior contribuição do metsulfuron para a eficácia da mistura, denotando sua maior capacidade para controle de dicotiledôneas, ou seja, neste caso, pode-se avaliar a interferência da mistura sobre a ação do metsulfuron-methyl. Sabidamente, a sintomatologia geral deste herbicida envolve amarelecimento da região meristemática com evolução para necrose e completa morte das plantas em 10 a 60 dias. Em aplicações pré-emergentes, a morte das plantas ocorre desde logo após a emergência até o estádio de duas folhas (Vidal et al., 2014).

Considerando-se concomitantemente as avaliações de controle e massa seca residual da corda-de-viola aos 14 e 28 DAA (Tabelas 3 e 4), somente a dose de $4,8 \mathrm{~g} \mathrm{ha}^{-1}$ de metsulfuron-methyl que garantiu a igualdade das doses de glyphosate. Para todas as outras doses de metsulfuron-methyl houve divergência entre as doses de glyphosate em ao menos uma avaliação (Tabelas 3 e 4). Neste ponto, a simples análise estatística tradicional permite concluir que a presença de metsulfuron--methyl na calda de pulverização incrementa os níveis de controle obtidos com o glyphosate sobre corda-de-viola, sobretudo para doses superiores a 3,6 $\mathrm{g} \mathrm{ha}^{-1}$.

Quanto à aplicação do método de Colby, para o capim-amargoso, detectou-se variabilidade geral dos dados, porém não foi identificado efeito negativo ou positivo da mistura de herbicidas (Tabela 5). Para tanto, nenhum dado observado foi diferente dos valores esperados, de forma que

Tabela 2. Massa seca residual ${ }^{1}$ do capim-amargoso (Digitaria insularis) após pulverização de diferentes doses do herbicida glyphosate e metsulfuron-methyl, isolados ou em mistura, avaliados aos 28 DAA. Machado - MG, 2015.

\begin{tabular}{ccccccc}
\hline $\begin{array}{c}\text { Glyphosate } \\
\left(\mathbf{g ~ h a}^{-1}\right)\end{array}$ & $\mathbf{0}$ & $\mathbf{1 , 2}$ & $\mathbf{2 , 4}$ & $\mathbf{3 , 6}$ & $\mathbf{4 , 8}$ & Média \\
\cline { 2 - 5 } & 6,24 & 5,36 & 6,27 & 4,35 & 5,41 & $5,53 \mathrm{D}$ \\
180 & 4,67 & 3,52 & 4,37 & 3,87 & 2,72 & $3,83 \mathrm{C}$ \\
360 & 2,69 & 2,99 & 2,20 & 2,73 & 2,51 & $2,63 \mathrm{~B}$ \\
540 & 1,64 & 1,92 & 1,48 & 2,20 & 1,95 & $1,84 \mathrm{~A}$ \\
720 & 1,73 & 1,27 & 1,50 & 1,51 & 1,50 & $1,50 \mathrm{~A}$ \\
Média & 3,39 & 3,01 & 3,16 & 2,93 & 2,82 & --- \\
& $\mathrm{CV}=16,12$ & $\mathrm{~F}_{\text {int }}=0,993^{\mathrm{NS}}$ & $\mathrm{F}_{\text {gly }}=42,519^{* *} \quad \mathrm{~F}_{\text {met }}=0,772^{\mathrm{NS}}$ & \\
\hline
\end{tabular}

${ }^{1}$ Médias seguidas por letras iguais, maiúsculas nas colunas e minúsculas nas linhas, não diferem entre si segundo teste de Scott-Knott, com 5\% de significância; Dados reais apresentados, porém previamente transformados por $\sqrt{x+1}$;

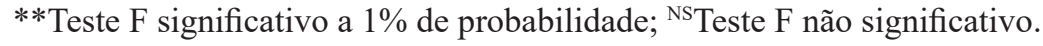


Tabela 3. Controle percentual ${ }^{1}$ da corda-de-viola (Ipomoea triloba) após pulverização de diferentes doses do herbicida glyphosate e metsulfuron-methyl, isoladas ou em mistura, avaliados aos 14 e 28 DAA. Machado - MG, 2015.

\begin{tabular}{|c|c|c|c|c|c|}
\hline \multirow{2}{*}{$\begin{array}{c}\text { Glyphosate } \\
\left(\mathrm{g} \mathrm{ha}^{-1}\right)\end{array}$} & \multicolumn{5}{|c|}{ Metsulfuron-methyl ( $\left.\mathrm{g} \mathrm{ha}^{-1}\right)$} \\
\hline & $\mathbf{0}$ & 1,2 & 2,4 & 3,6 & 4,8 \\
\hline \multicolumn{6}{|c|}{ Avaliação de controle aos 14 dias após aplicação } \\
\hline 0 & $0,0 \mathrm{~B} \mathrm{a}$ & $41,5 \mathrm{~B} \mathrm{~b}$ & $46,7 \mathrm{~A} \mathrm{a}$ & $50,8 \mathrm{~A} \mathrm{a}$ & $49,8 \mathrm{~A} \mathrm{a}$ \\
\hline 180 & $41,8 \mathrm{~A} \mathrm{a}$ & $46,0 \mathrm{~B} \mathrm{a}$ & $50,0 \mathrm{~A} \mathrm{a}$ & $45,7 \mathrm{~B} \mathrm{a}$ & $48,0 \mathrm{~A} \mathrm{a}$ \\
\hline 360 & $47,8 \mathrm{~A} \mathrm{a}$ & $45,3 \mathrm{~B}$ a & $48,7 \mathrm{~A} \mathrm{a}$ & $52,8 \mathrm{~A} \mathrm{a}$ & $45,5 \mathrm{~A} \mathrm{a}$ \\
\hline 540 & $47,5 \mathrm{~A} \mathrm{~b}$ & $53,5 \mathrm{~A} \mathrm{a}$ & $50,3 \mathrm{~A} \mathrm{a}$ & $45,7 \mathrm{~B} \mathrm{~b}$ & $51,2 \mathrm{~A} \mathrm{a}$ \\
\hline 720 & $44,5 \mathrm{~A} \mathrm{~b}$ & $48,7 \mathrm{~A} \mathrm{~b}$ & $53,8 \mathrm{~A} \mathrm{a}$ & $47,0 \mathrm{~B} \mathrm{~b}$ & $47,0 \mathrm{~A} \mathrm{~b}$ \\
\hline & $\mathrm{CV}=10,94$ & $\mathrm{~F}_{\mathrm{int}}=20,555^{* *}$ & $\mathrm{~F}_{\mathrm{olv}}=27,002 * *$ & $\mathrm{~F}_{\mathrm{met}}=36,006^{* *}$ & \\
\hline \multicolumn{6}{|c|}{ Avaliação de controle aos 28 dias após aplicação } \\
\hline 0 & $0,0 \mathrm{C} \mathrm{b}$ & $47,3 \mathrm{~B} \mathrm{a}$ & $53,5 \mathrm{~B} \mathrm{a}$ & $51,3 \mathrm{~B} \mathrm{a}$ & $60,3 \mathrm{~A} \mathrm{a}$ \\
\hline 180 & $44,3 \mathrm{~B} \mathrm{~b}$ & $51,7 \mathrm{~B} \mathrm{~b}$ & $68,2 \mathrm{~A} \mathrm{a}$ & $69,5 \mathrm{~A} \mathrm{a}$ & $67,8 \mathrm{~A} \mathrm{a}$ \\
\hline 360 & $57,2 \mathrm{~A} \mathrm{~b}$ & $52,2 \mathrm{~B} \mathrm{~b}$ & $69,7 \mathrm{~A} \mathrm{a}$ & $66,8 \mathrm{~A} \mathrm{a}$ & $70,3 \mathrm{~A} \mathrm{a}$ \\
\hline 540 & $55,5 \mathrm{~A} \mathrm{~b}$ & $67,7 \mathrm{~A} \mathrm{a}$ & $60,3 \mathrm{~B} \mathrm{~b}$ & $68,5 \mathrm{~A} \mathrm{a}$ & $70,0 \mathrm{~A} \mathrm{a}$ \\
\hline \multirow[t]{2}{*}{720} & $55,8 \mathrm{~A} \mathrm{~b}$ & $70,3 \mathrm{~A} \mathrm{a}$ & $75,8 \mathrm{~A} \mathrm{a}$ & $76,7 \mathrm{~A} \mathrm{a}$ & $75,3 \mathrm{~A} \mathrm{a}$ \\
\hline & $\mathrm{CV}=15,58$ & $\mathrm{~F}_{\mathrm{int}}=5,575^{* *}$ & $\mathrm{~F}_{\mathrm{gly}}=38,514 * *$ & $\mathrm{~F}_{\mathrm{met}}=39,029 * *$ & \\
\hline
\end{tabular}

${ }^{1}$ Médias seguidas por letras iguais, maiúsculas nas colunas e minúsculas nas linhas, não diferem entre si segundo teste de Scott-Knott, com 5\% de significância; **Teste F significativo a 1\% de probabilidade.

Tabela 4. Massa seca residual ${ }^{1}$ da corda-de-viola (Ipomoea triloba) após pulverização de diferentes doses do herbicida glyphosate e metsulfuron-methyl, isolados ou em mistura, avaliados aos 28 DAA. Machado - MG, 2015.

\begin{tabular}{ccccccc}
\hline $\begin{array}{c}\text { Glyphosate } \\
\left(\mathbf{g ~ h a}^{-1}\right)\end{array}$ & $\mathbf{0}$ & $\mathbf{1 , 2}$ & $\mathbf{2 , 4}$ & $\mathbf{3 , 6}$ & \multirow{2}{*}{ Metsuldia } \\
\cline { 2 - 5 } & $2,45 \mathrm{C} \mathrm{b}$ & $0,80 \mathrm{~B} \mathrm{a}$ & $0,75 \mathrm{~B} \mathrm{a}$ & $0,70 \mathrm{~A} \mathrm{a}$ & $0,53 \mathrm{~A} \mathrm{a}$ & 1,05 \\
180 & $1,10 \mathrm{~B} \mathrm{~b}$ & $0,73 \mathrm{~B} \mathrm{a}$ & $0,48 \mathrm{~A} \mathrm{a}$ & $0,48 \mathrm{~A} \mathrm{a}$ & $0,51 \mathrm{~A} \mathrm{a}$ & 0,66 \\
360 & $0,78 \mathrm{~A} \mathrm{~b}$ & $0,79 \mathrm{~B} \mathrm{~b}$ & $0,49 \mathrm{~A} \mathrm{a}$ & $0,52 \mathrm{~A} \mathrm{a}$ & $0,53 \mathrm{~A} \mathrm{a}$ & 0,62 \\
540 & $0,86 \mathrm{~A} \mathrm{~b}$ & $0,51 \mathrm{~A} \mathrm{a}$ & $0,55 \mathrm{~A} \mathrm{a}$ & $0,44 \mathrm{~A} \mathrm{a}$ & $0,43 \mathrm{~A} \mathrm{a}$ & 0,56 \\
720 & $0,62 \mathrm{~A} \mathrm{a}$ & $0,51 \mathrm{~A} \mathrm{a}$ & $0,41 \mathrm{~A} \mathrm{a}$ & $0,44 \mathrm{~A} \mathrm{a}$ & $0,40 \mathrm{~A} \mathrm{a}$ & 0,48 \\
Média & 1,16 & 0,67 & 0,54 & 0,52 & 0,48 & -- \\
& $\mathrm{CV}=6,04$ & $\mathrm{~F}_{\text {int }}=8,413^{* *}$ & $\mathrm{~F}_{\text {gly }}=27,958^{* *} \quad \mathrm{~F}_{\text {met }}=47,104^{* *}$ & \\
\hline
\end{tabular}

${ }^{1}$ Médias seguidas por letras iguais, maiúsculas nas colunas e minúsculas nas linhas, não diferem entre si segundo teste de Scott-Knott, com 5\% de significância; Dados reais apresentados, porém previamente transformados por $\sqrt{x+1}$; $* *$ Teste $\mathrm{F}$ significativo a $1 \%$ de probabilidade.

Tabela 5. Análise da interação das misturas de glyphosate e metsulfuron-methyl aos 28 dias após pulverização sobre o capim-amargoso (Digitaria insularis). DMS ${ }_{5 \%}=19,47$. Machado - MG, 2015.

\begin{tabular}{|c|c|c|c|c|c|c|c|c|c|c|c|c|}
\hline \multirow{3}{*}{$\begin{array}{l}\text { Glyphosate } \\
\left(\mathrm{g} \mathrm{ha}^{-1}\right)\end{array}$} & \multicolumn{12}{|c|}{ Metsulfuron-methyl ${ }^{1}\left(\mathrm{~g} \mathrm{ha}^{-1}\right)$} \\
\hline & \multicolumn{3}{|c|}{1,2} & \multicolumn{3}{|c|}{2,4} & \multicolumn{3}{|c|}{3,6} & \multicolumn{3}{|c|}{4,8} \\
\hline & Obs. ${ }^{1}$ & Esp. $^{2}$ & Int. $^{3}$ & Obs. ${ }^{1}$ & Esp. $^{2}$ & Int. $^{3}$ & Obs. ${ }^{1}$ & Esp. $^{2}$ & Int. $^{3}$ & Obs. ${ }^{1}$ & Esp. $^{2}$ & Int. $^{3}$ \\
\hline 180 & 51,3 & 45,7 & $=$ & 29,8 & 43,2 & $=$ & 39,3 & 50,4 & $=$ & 65,8 & 46,4 & $=$ \\
\hline 360 & 53,3 & 71,0 & $=$ & 82,2 & 69,7 & $=$ & 76,3 & 73,5 & $=$ & 76,5 & 71,4 & $=$ \\
\hline 540 & 88,2 & 92,6 & $=$ & 95,5 & 92,2 & $=$ & 88,2 & 93,2 & $=$ & 88,2 & 92,7 & $=$ \\
\hline 720 & 99,5 & 96,4 & $=$ & 97,2 & 96,3 & $=$ & 98,0 & 96,7 & $=$ & 98,2 & 96,5 & $=$ \\
\hline
\end{tabular}

${ }^{1}$ Valores observados; ${ }^{2}$ Valores esperados; ${ }^{3}$ Análise da interação, considerando-se teste ' $t$ ' aplicado com $5 \%$ de significância, em que $(+)$ diz respeito à mistura sinérgica, $(=)$ diz respeito à mistura aditiva e $(-)$ diz respeito à mistura antagônica. 
Tabela 6. Análise da interação das misturas de glyphosate e metsulfuron-methyl aos 7 e 14 dias após pulverização sobre a corda-de-viola (Ipomoea triloba). DMS $\mathrm{DM}_{5 \%}=10,73$. Machado - MG, 2015.

\begin{tabular}{|c|c|c|c|c|c|c|c|c|c|c|c|c|}
\hline \multirow{3}{*}{$\begin{array}{l}\text { Glyphosate } \\
\left(\mathrm{g} \mathrm{ha}^{-1}\right)\end{array}$} & \multicolumn{12}{|c|}{ Metsulfuron-methyl ${ }^{1}\left(\mathrm{~g} \mathrm{ha}^{-1}\right)$} \\
\hline & \multicolumn{3}{|c|}{1,2} & \multicolumn{3}{|c|}{2,4} & \multicolumn{3}{|c|}{3,6} & \multicolumn{3}{|c|}{4,8} \\
\hline & Obs. ${ }^{1}$ & Esp. ${ }^{2}$ & Int. $^{3}$ & Obs. $^{1}$ & Esp. ${ }^{2}$ & Int. $^{3}$ & Obs. ${ }^{1}$ & Esp. $^{2}$ & Int. $^{3}$ & Obs. ${ }^{1}$ & Esp. ${ }^{2}$ & Int. $^{3}$ \\
\hline 180 & 51,7 & 70,6 & - & 68,2 & 74,1 & $=$ & 69,5 & 72,9 & $=$ & 67,8 & 77,9 & $=$ \\
\hline 360 & 52,2 & 77,4 & - & 69,7 & 80,1 & $=$ & 66,8 & 79,2 & - & 70,3 & 83,0 & - \\
\hline 540 & 67,7 & 76,5 & $=$ & 60,3 & 79,3 & - & 68,5 & 78,3 & $=$ & 70,0 & 82,3 & - \\
\hline 720 & 70,3 & 76,7 & $=$ & 75,8 & 79,4 & $=$ & 76,7 & 78,5 & $=$ & 75,3 & 82,5 & $=$ \\
\hline
\end{tabular}

${ }^{1}$ Valores observados; ${ }^{2}$ Valores esperados; ${ }^{3}$ Análise da interação, considerando-se teste ' $t$ ' aplicado com $5 \%$ de significância, em que $(+)$ diz respeito à mistura sinérgica, $(=)$ diz respeito à mistura aditiva e (-) diz respeito à mistura antagônica.

não houve evidências de efeitos sinérgicos ou antagônicos para esta mistura, considerando-se o controle da espécie monocotiledônea. Para todas as comparações, as misturas foram consideradas aditivas (Tabela 5), embora haja trabalhos na literatura científica que relatam a tendência de sinergismo da mistura (Kudsk e Mathiassem, 2003).

Neste sentido, Alcântara e Silva (2002) comentam que esta mistura amplia o espectro de controle do glyphosate, além de permitir efeito residual do tratamento. Arizaleta et al. (2008), também estudando o efeito da mistura de glyphosate e metsulfuron-methyl, constataram redução satisfatória na densidade de plantas daninhas. De fato, maior controle absoluto foi observado para corda-de-viola, porém numericamente aquém dos valores esperados, obtidos pela equação (Tabela 6).

Assim sendo, as análises de interação para corda-de-viola resultaram em observações divergentes em relação ao capim-amargoso (Tabela 6), bem como da hipótese científica. Neste caso, dentre as 16 combinações de doses avaliadas, os valores de controle observados foram inferiores aos valores esperados em seis combinações, com diferença maior que a DMS do teste ' $t$ ', indicando antagonismo da mistura. Esse resultado se opõe os obtidos por Inoue et al. (2012), que verificaram eficácia da mistura na dessecação de plantas adultas de Crotalaria (Crotalaria spectabilis).
Neste sentido, ressalta-se que o método de Colby (1967) é muito rigoroso e só considera sinergismo os casos em que a mistura resulta em eficácia observada significativamente superior à esperada, o que não ocorreu para os bioindicadores avaliados. Ou seja, mesmo na ocorrência de valores de controle numericamente superiores às doses isoladas, estes deveriam ser ainda de maior magnitude para denotar uma mistura sinérgica.

\section{Conclusão}

A aplicação da mistura de glyphosate e metsulfuron-methyl sobre o capim-amargoso foi considerada aditiva em todas as doses utilizadas; já sobre a corda-de-viola, a adição de metsulfuron-methyl à calda de glyphosate eleva o controle absoluto da mistura, porém com magnitude aquém da esperada, o que denota caráter antagônico da mistura.

\section{Agradecimentos}

Os autores agradecem à Fundação de Amparo à Pesquisa de Minas Gerais - FAPEMIG pela bolsa de iniciação científica concedida ao primeiro autor.

\section{Referências}

Alcântara, E.N.; Silva, F.M.A. Controle de plantas daninhas em cafeeiros com metsulfuron e em mistura com glyphosate. In: Congresso Brasileiro da Ciência das Plantas Daninhas, 23., 
2002, Londrina. Resumos... Londrina: SBCPD/ Embrapa Clima Temperado, 2002. p.477.

Arizaleta, M.; Anzalone, A.; Silva, A. Efecto del uso de metsulfuron-metil y glifosato sobre malezas associados a cafetales en Venezuela. Bioagro-, v.20, n.2, p.79-88, 2008.

Barroso, A.A.M.; Albrecht, A.J.P.; Reis, F.C.; Victoria Filho, R. Interação entre herbicidas inibidores da ACCase e diferentes formulações de glyphosate no controle de capim-amargoso. Planta Daninha, v.32, n.3, p.619-627, 2014.

Carbonari, C.A.; Martins, D.; March, S.R.; Cardoso, L.R. Efeito de surfactantes e pontas de pulverização na deposição de calda de pulverização em plantas de grama-seda. Planta Daninha, v.23, n.4, p.725-729, 2005.

Christoffoleti, P.J.; Galli, A.J.B.; Carvalho, S.J.P.; Moreira, M.S.; Nicolai, M.; Foloni, L.L.; et al. Glyphosate sustainability in South American cropping systems. Pest Management Science, v.64, n.4, p.422-427, 2008.

Ciuberkis, S.; Bernotas, S.; Raudonis, S.; Felix, J. Effect of weed emergence time and intervals of weed and crop competition on potato yield. Weed Technology, v.21, n.1, p.213-218, 2010.

Colby, S.R. Calculating synergistic and antagonistic responses of herbicide combinations. Weeds, v.15, n.1, p.20-22, 1967.

Gazziero, D.L.P. Misturas de agrotóxicos em tanque nas propriedades agrícolas do Brasil. Planta Daninha, v.33, n.1, p.83-92, 2015.

Inoue, M.H.; Duarte, J.C.B.; Mendes, K.F.; Sztoltz, J.; Ben, R.; Pereira, R.L. Eficácia de herbicidas aplicados em plantas adultas de Crotalaria spectabilis e Crotalaria ochroleuca. Revista Brasileira de Herbicidas, v.11, n.2, p.148-158, 2012.

Kudsk, P.; Mathiassem, S.K. Joint action of amino acid biosynthesis-inhibiting herbicides. Weed Research, v.44, n.4, p.313-322, 2003.
Lich, J.M.; Renner, K.A.; Penner, D. Interaction of glyphosate with postemergence soybean (Glycine max) herbicides. Weed Science, v.45, n.1, p.12-21, 1997.

Maciel, C.D.G.; Poletine, J.P.; Amstalden, S.L.; Gazziero, D.L.P.; Raimond, M.A.; Lima, G.R.G.; et al. Misturas em tanque com glyphosate para o controle de trapoeraba, erva-de-touro e capim-carrapicho em soja $\mathrm{RR} \AA$. Revista Ceres, v.58, n.1, p.35-42, 2011.

Moldes, C.A.; Camiña, J.M.; Medici, L.O.; Tsai, S.M.; Azevedo, R.A. Physiological effects of glyphosate over amino acid profile in conventional and transgenic soybean (Glycine max). Pesticide Biochemistry and Physiology, v.102, n.2, p.134141, 2012.

Moreira, M.S.; Melo, M.S.C.; Carvalho, S.J.P.; Nicolai, M.; Christoffoleti, P.J. Herbicidas alternativos para controle de biótipos de Conyza bonariensis e C. canadensis resistente ao glyphosate. Planta Daninha, v.28, n.1, p.167-175, 2010.

Peterson, D.E. The impact of herbicide-resistant weeds on Kansas agriculture. Weed Technology, v.13, n.3, p.632-635, 1999.

Ramires, A.C.; Constantin, J.; Oliveira Junior, R.S.; Guerra, N.; Alonso, D.G.; Biffe, D.F. Control of Euphorbia heterophylla and Ipomoea grandifolia using glyphosate isolated or in association with broadleaf herbicides. Planta Daninha, v.28, n.3, p.621-629, 2010.

Rodrigues, B.N.; Almeida, F.S. Guia de Herbicidas. 6.ed. Londrina: Grarfmake, 2011. 697p.

SBCPD - SOCIEDADE BRASILEIRA DA CIÊNCIA DAS PLANTAS DANINHAS. Procedimentos para instalação, avaliação e análise de experimentos com herbicidas. Londrina: SBCPD, 1995. 42p.

Trezzi, M.M.; Diesel, F.; Kruse, N.D.; Xavier, E.; Pazuch, D.; Pagnoncelli Junior, F.; Batistel, S.C. Interactions of saflufenacil with other herbicides promoters of oxidative stress to 
control joyweed. Planta Daninha, v.34, n.2, p.319-326, 2016.

Vargas, L.; Peixoto, C.M.; Roman, E.S. Manejo de plantas daninhas na cultura do milho. Passo Fundo: Embrapa Trigo, 2006.20p. Documentos 61.
Vidal, R.A.; Merotto Junior, A.; Schadler, C.E.; Lamego, F.P.; Portugal, J.; Mendes, J.; et al. Mecanismos de ação dos herbicidas. In: Monquero, P.A. (Coord.). Aspectos da biologia e manejo das plantas daninhas. São Carlos: RiMa, 2014. p. 235-256. 